\title{
Abemaciclib/Anastrozole Regimen
}

National Cancer Institute

\section{Source}

National Cancer Institute. Abemaciclib/Anastrozole Regimen. NCI Thesaurus. Code C156379.

A chemotherapy regimen consisting of abemaciclib and anastrozole that may be used as a treatment for hormone receptor (HR)-positive breast cancer. 